\title{
P133: The practice of hand hygiene among manicures and pedicures in Brazil
}

\author{
JL Garbaccio, AC Oliveira, AO de Paula* \\ From 2nd International Conference on Prevention and Infection Control (ICPIC 2013) \\ Geneva, Switzerland. 25-28 June 2013
}

\section{Introduction}

Hand hygiene is the primary mechanism to control the dissemination of microorganisms. Manicures and pedicures touch the hands and the feet of customers that can be very contaminated.

\section{Objectives}

This study evaluated the practice of hand hygiene of manicures/pedicures in Brazil.

\section{Methods}

This Survey included a random sample of 200 professionals older than 18 , covering 200 beauty salons of Belo Horizonte, in Brazil. A questionnaire was used (Jul/ 12-Jan/13) to obtain information of demographics and their knowledge about the actual practice of hand washing. The results were analyzed by the statistical program SPSS. The study was approved by the Ethics Committee of the Federal University of Minas Gerais.

\section{Results}

All professionals interviewed were women with an average age of 30 , more than 10 years of experience $(11 \%)$, working more than 8 hours per day (57\%), less than a year in that salon (34\%). As for education $55 \%$ had completed high school, $54 \%$ had done some training course in the field, yet $38 \%$ became manicure/pedicure by her own initiative. Moreover, $76 \%$ had never received any training in biosafety. From the knowledge questions, almost all of the respondents (99\%) said it was important to wash their hands between customers schedules, using liquid soap stored in dispensers $(81 \%)$ and dry them with a disposable paper towel (86\%). However, $34 \%$ reported that they were not washing their hands, $14 \%$ using bar soap and $17 \%$ used a tissue towel without a daily change. The main reason for not joining the practice was the lack of time between appointments. There was no statistically significant association between demographics and the practice of hand washing ( $p>0.05$ ). We evaluated the use of accessories like rings, bracelets and watches as well as the length of the nails of the professionals. More than half (53\%) reported not removing the accessories, but on the day of the interview, $60 \%$ were found to be using at least one. The nails of $44 \%$ of the manicures/pedicures were long despite $38 \%$ stated that they keep them short.

\section{Conclusion}

It was concluded that educational actions for manicures/ pedicures should be implemented to encourage them to adhere to the hand washing practice that is so important for their own health and that of their customers'.

\section{Funding agent}

Fundação de Amparo a Pesquisa de Minas Gerais. Programa Pesquisador Mineiro $n^{\circ}$ 00340-11

\section{Disclosure of interest}

None declared.

Published: 20 June 2013

doi:10.1186/2047-2994-2-S1-P133

Cite this article as: Garbaccio et al:: P133: The practice of hand hygiene among manicures and pedicures in Brazil. Antimicrobial Resistance and Infection Control 2013 2(Suppl 1):P133.

Nursing, Federal University of Minas Gerais - Brazil, Belo Horizonte, Brazil 